\title{
Could OKAN be an objective indicator of the susceptibility to visually induced motion sickness?
}

\author{
Cuiting Guo, Jennifer Ji, Richard So \\ Department of Industrial Engineering and Logistics Management, Hong Kong University of Science and Technology
}

\begin{abstract}
International Workshop Agreement 3 organized by the International Standard Organization calls for more research to determine simple objective ways to assess susceptibility to visually induce motion sickness (VIMS) without making viewers sick (So and Ujike, 2010). This study examines the use of measurable optokinetic afternystagmus (OKAN) parameters to predict susceptibility to VIMS. Eighteen participants were recruited. They were exposed to a sickness provoking virtual rotating drum (210 degrees field-of-view) with striped patterns rotating at 60 degrees per second for 30 minutes (Phase 1). Sickness data were collected before, during, and after the exposure. These participants were invited back for OKAN measurements at least two weeks after Phase 1 was completed to minimize any adaption effect (Phase 2). Out of the 18 participants, 10 participants (i.e., 55\%) exhibited consistent patterns of OKAN. Correlations between the time constants of OKAN and levels of VIMS experienced by the same viewers were found. The possibility of using OKAN as an objective indicator of the susceptibility to visually induced motion sickness is discussed.
\end{abstract}

KeYWORDS: OKAN, Visually induced motion sickness, Velocity storage mechanism, Motion sickness.

INDEX TERMS: simulation and behavior; psychology; immersion; cybersickness

\section{INTRODUCTION}

Watching striped patterns rotating in the yaw axis can trigger optokinetic nystagmus (OKN) and visually induced motion sickness (VIMS) (Ebenholtz, 1992; Ji et al., 2009; Lo and So, 2001). About $30 \%$ of the British and Chinese population are susceptible to motion sickness (Griffin, 1992; So et al., 1999) and about $30 \%$ of viewers of virtual reality (VR) simulation are susceptible to VIMS (Lo and So, 2001; Kiryu and So, 2008; So et al., 2001). A typical OKN cycle consists of a slow drift of the eyes in the direction of the stimuli followed by a rapid saccadic return. While viewers are experiencing $\mathrm{OKN}$, a sudden immersion into total darkness will stop OKN in some viewers and allow slow decays of $\mathrm{OKN}$ in others. The latter is called optokinetic afternystagmus (OKAN). It is a continuation of OKN but with a slowly decaying drifting velocity (Ohm, 1927). OKAN occurs in the same direction as OKN. Sometimes it is followed by a

Department of Industrial Engineering and Logistics Management, Hong Kong University of Science and Technology, Clearwater Bay, Kowloon, Hong Kong.

Email: rhyso@ust.hk secondary afternystagmus (OKAN II) beating in the opposite direction to OKN (Aschan and Bergstedt, 1955). If OKN response can be divided into the visual perception part and the vestibular response part, OKAN should reflect the part involving the activation of the vestibular nucleus (Tijssen et al., 1989). It has been known that patients with bilateral labyrinthectomy will not experience OKAN (Cohen et al., 1973) and OKAN can be suppressed after surgical removal of the flocculi and portions of the paraflocculi within the vestibular organ (Waespe, 1983). Dellepiane et al. (2006) reported that the prevalence rate of OKAN increased with increasing vestibular reflex in individuals. This suggests that the vestibular system plays an important role in the genesis of OKAN.

On the other hand, the vestibular nuclei have also been reported to play a central role in the generation of visually induced motion sickness (VIMS). Cheung et al. (1991) reported that bilateral labyrinthine-defective subjects were immune from VIMS but can experience vection sensation (illusion of self-motion). A review of literature indicate that self-report questionnaires have been the most common way to measure levels of VIMS (e.g., Kennedy et al., 1993; Ji et al., 2009; Lo and So, 2001; So and Lo, 1999; So et al., 2002). Although gastric myoelectric activities have been shown to correlate with rated levels of visually induced motion sickness, its measurement requires making the participants sick (Hu et al., 1989). In 2005, ISO International Workshop Agreement 3 (IWA3) called for the development of simple objective tests to assess susceptibility to VIMS. Consequently, a study has been conducted to investigate whether OKAN can be an objective measure for quantifying susceptibility to VIMS (So and Ujike, 2010).

OKAN is known to be a reflection of the velocity storage mechanism (VSM). There are two pathways related to the generation of OKN and OKAN. A direct pathway linked to the smooth pursuit system is responsible for the rapid rise of OKN and rapid release of OKAN (Muratore and Zee, 1979). The secondary slow rise and steady-state of OKN slow phase velocity and the slow decay of OKAN slow phase velocity is mediated by an indirect vestibular pathway. The direct pathway is also known as the fast pathway and the indirect pathway is known as the slow pathway. During optokinetic stimulation, the indirect component would engage eye velocity signals to contribute to the build-up of OKN velocity. When in total darkness, the discharge of the eye velocity storage would maintain the eye response, resulting in OKAN. This has been referred to as the velocity storage mechanism (VSM). VSM can be trigged either by visual or vestibular stimulus, which can be reflected by the time course of OKAN or the vestibo-ocular reflex (VOR).

The authors of this paper are not the first to link OKAN with motion sickness. When testing patients with labyrinthine lesions, Dai et al. (2003) reported that susceptibility to physical motion sickness is related to the indirect vestibular pathway (also known as the slow pathway) known to control the steady-state of OKN slow phase velocity and its decline during OKAN. Perhaps the strongest evidence reported so far is the ability of 'Baclofen' (a 
drug) to increase the activities of GABA receptors resulting in shortening of the time constant of velocity decay in OKAN as well as preventing symptoms of physical motion sickness in specific forms of vertigo (Dai et al., 2005). We, therefore, hypothesized in this study that, the slow phase velocity decay time constants of OKAN correlates with individual susceptibility to VIMS (H1). This hypothesis is also consistent with past studies on vestibular ocular reflex (VOR). Significant correlations between motion sickness susceptibility and the angular VOR time constants have been reported and it has been suggested that motion sickness is related to the indirect pathway (Dai et al., 2007; Takahashi et al., 1997).

Further literature reviews indicate that studies to relate OKAN and VIMS on normal human beings are not yet available.

\section{METHOD}

Nine male and nine female subjects were recruited for the experiment. They were exposed to a virtual rotating drum which has previously been used to provoke VIMS and postural instability among viewers (Ji et al., 2009; Chow et al., 2007). Figure 1 illustrates the virtual rotating drum. During this phase (Phase 1), participants were required to watch alternate black-andwhite vertical stripes rotating clockwise at 60 degrees per second for 30 minutes while standing. A chin-rest was used to restrain head movements. In addition, the head positions of participants were monitored using a Polhemus FASTRAK sensor. The display was updated at 60 frames per second. A previous study indicates that additional display lag of $40 \mathrm{~ms}$ may affect subject ratings (So and Griffin, 1991a,b, 1992). In this experiment, images were projected on a curved screen and their projection onto viewers' retinas response instantly to eye movements. Symptoms of motion sickness were measured before, during, and after the exposure using a pre-exposure simulator sickness questionnaire (SSQ), a 7point nausea rating (Golding, 2006), and a post-exposure SSQ. The slow phase velocities of OKNs were measured using an EOG system (EOG 100C from BIAPAC ${ }^{\circledR}$ ).

Two weeks after the exposure in Phase 1, participants were invited back for Phase 2 of the study. They were exposed to the same virtual rotating drum for six repeated trials. The six repetitions were separated into two consecutive days to minimize eye fatigue. Phases 1 and 2 shared the same laboratory environment and apparatus.

Each trial consisted of three stages: (i) participants were instructed to stare at a stationary fixation dot projected on a blank screen for 15 seconds; (ii) after the 15 -second period, the virtual drum was started and rotated at 60 degrees per second in the clockwise direction for two minutes. During this period, participants were instructed to look straight ahead; (iii) on the 120th second, the stimulus as well as all light in the laboratory were switched off for one minute and participants were instructed to keep looking straight ahead during this period.

Eye movement data was recorded by the EOG system (EOG100C, BIAPAC®). Two commonly used parameters of
OKAN were analyzed (Dai et al., 2003): the initial velocity of OKAN and the time constant of OKAN. Data corresponding to the first second (i.e., the $120^{\text {th }}$ second) after the light was switched off was discarded following the literature (Tijssen et al., 1989).

The initial velocity of OKAN was calculated from the EOG data collected during the 2nd second after the light was switched off (i.e., the $121^{\text {st }}$ second). Most participants had more than one OKAN cycle during the $121^{\text {st }}$ second. For those who had only one cycle, the first OKAN cycle was used to calculate the initial velocity of OKAN. The decline of the slow phase velocity was modelled with an exponential function (Cohen et al., 1977, Muratore and Zee, 1979, Maioli, 1988, and Tijssen et al., 1989). The time constant was determined by the reciprocal of the slope of the fitted line to plots of the natural $\log (\ln )$ of the slow phase velocity versus the time of exposure to total darkness.

\section{Results}

\subsection{Head Movements}

The average standard deviations of the lateral head movements of the eighteen participants ranged from $0.02 \mathrm{~cm}$ to $0.32 \mathrm{~cm}$. The data indicate that participants' heads were well restrained by the chin rest.

\subsection{Prevalence Rates of OKAN}

The prevalence rate of OKAN among the 18 participants was $55 \% .10$ out of 18 participants exhibited patterns of OKAN. There was no significant correlation between the occurrence of OKAN and the rated level of nausea reported in Phase 1 of the study. Figures 2 and 3 are examples of consistent occurrence and

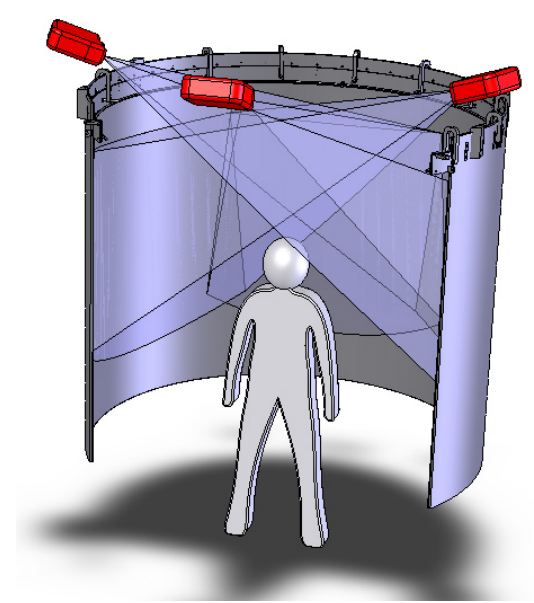

Figure 1. An illustration of a viewer facing the virtual rotating drum with field-of-view of $210^{\circ}$ (horizontal) by $50^{\circ}$ (vertical).

Table 1. Tally of OKAN occurrence among the 18 participants

\begin{tabular}{|c|c|c|c|}
\hline Consistently present & \multicolumn{2}{|c|}{ Inconsistently/Occasionally present } & Consistently absent \\
\hline All 6 trials & 5 out of 6 trials & 1 to 4 trials & $\begin{array}{c}0 \% \text { (as in "None of the } \\
\text { trials"?) }\end{array}$ \\
\hline 5 & 2 & 3 & 8 \\
\hline
\end{tabular}


consistent absence of OKAN respectively. As shown in Table 1, only 5 out of the 18 participants showed consistent occurrence of OKAN in all six trials.

\subsection{Initial Slow Phase Velocity of OKAN}

Among the eighteen participants, eight participants did not show patterns of OKAN. The average 7-point nausea rating of these eight participants was 3.82 , ranging from 1.5 to 5.8 , while the average 7-point nausea rating for the ten participants who exhibited OKAN was 3.95, ranging from 1 to 5.8. The average 7point nausea rating for all 18 participants was 3.85 . No observable difference in susceptibility to VIMS was found between the two groups of participants.

The average initial velocity of all ten subjects who exhibited OKAN was $7.9 \mathrm{dps}$, ranging from $3.8 \mathrm{dps}$ to $11.5 \mathrm{dps}$. This represented a reduction of $80 \%$ in slow phase velocity from OKN to OKAN. There was no observable difference in initial velocities between male and female participants.

Correlation tests were conducted between average nausea ratings and the initial velocities of OKAN. There was no significant correlation between the susceptibility to motion sickness and the initial velocity of OKAN (Pearson correlation: $\rho=-0.365, p=0.05$ ). The discharge rates (initial SPV / SPV of $\mathrm{OKN}$ ) were calculated for each trial and there was no significant correlation between the rated levels of nausea in Phase 1 and the discharge rates $(\rho=0.121, p>0.05)$.

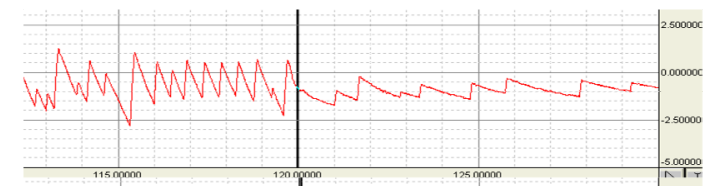

Figure 2. An example of a viewer showing consistent occurence of OKAN. The horizontal axis is time and the vertical axis is the voltage of EOG signals. OKN patterns can be observed before the light was turned off (indicated by the vertical black line). OKAN patterns can be observed after the light was turned off (adapted from Guo et al., 2011).

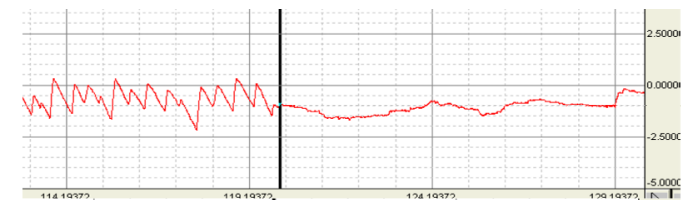

Figure 3. An example of a viewer showing consistent absence of OKAN. The horizontal axis is time and the vertical axis is the voltage of EOG signals. OKN patterns can be observed before the light was turned off (indicated by the vertical black line). No OKAN patterns can be observed after the light was turned off (adapted from Guo et al., 2011)

\subsection{Time Constants of the Decaying OKAN velocity}

The decaying curve of OKAN velocity was modelled by an exponential function (Tijsen et al., 1989). Linearity correlation between the logarithm of the slow phase velocity of OKAN and duration in each trial was tested before fitting a linear regression line. Those showing no significant linearity were discarded in the calculation of time constants which were the slopes of the fitted lines. All except one trial reported significant linear correlation $(p<0.05)$. The one that did not show a significant correlation was due to a very short duration of OKAN.

The average time constant of the ten participants who exhibited OKAN patterns was $17.9 \mathrm{~s} \pm 5 \mathrm{~s}$, ranging from $6.7 \mathrm{~s}$ to $30 \mathrm{~s}$; for male subjects it was $16.8 \mathrm{~s}$ and for female subjects it was $18.81 \mathrm{~s}$. There was no significant difference between the time constants of male and female participants. Pearson correlation tests showed that there was marginally significant correlation between the time constants and the rated levels of nausea reported in Phase 1 of the study $(p=0.05$, correlation coefficient: $\rho=0.484)$. This is an important and interesting finding which extends Dai et al. (2003)'s result to visually induced motion sickness. The authors of this paper are delighted to have found significant results even with simple forms of data extraction. An inspection of Figures 1, 2 and 3 indicates that OKAN cycles are nosy. More sophisticated data smoothing techniques may result in better signal to noise ratio. The authors are currently consulting signal processing experts about the best way to detect and extract OKAN data.

The authors also used the intercepts of the fitted regression lines as an alternative method to determine the initial slow phase velocity of OKAN. The initial velocities calculated using this method significantly correlated with those calculated using just the $121^{\text {st }}$ second data $(\rho=0.8, \mathrm{p}<0.001)$. This supports the validity of the fitted regression lines in modelling the slow phase velocity of OKAN. .

\section{DISCUSSION AND CONCLUSION}

This study examined the correlation of OKAN parameters with rated levels of VIMS on normal human participants. Eighteen participants were studied and ten of them exhibited patterns of OKAN. The initial slow phase velocity and time constant of OKAN were characterized. Based on the statistical results, there are two major findings. First, motion sickness susceptibility, as indicated by the rated levels of nausea in Phase 1 of this study, significantly correlated with the time constants of decaying OKAN slow phase velocity. Since the decaying process is related to the indirect velocity storage pathway, the significant correlation suggests that the susceptibility of VIMS may also be related to the indirect velocity storage pathway. Second, motion sickness susceptibility is independent of the direct pathway. This was indicated by the non-significant correlation between the initial slow phase velocity of OKAN and the rated levels of nausea and the non-significant correlation between the discharge rate of OKAN and the rated level of nausea. These two findings are consistent with past studies concerning the correlation relationships between vetibulo-ocular reflex (VOR) and susceptibility to motion sickness. Susceptibility to physical motion sickness have been shown to be related to the indirect pathway (significantly correlated with angular VOR time constants: Dai et al., 2007) but not to the direct pathway (nonsignificant correlation with angular VOR gains: Cohen et al., 2003; Dai et al., 2003; 2007). The authors would like to stress that most/all previous studies have been focused on physical motion sickness and no study relating OKAN time constants to levels of VIMS could be found.

The current findings indicate that OKAN measurement is/can be a quick and simple test to measure individuals' susceptibility to visually induced motion sickness without making them sick. However, this study only involved 18 participants. Future studies with more participants are desirable to confirm the findings. Also, the requirement of a complete dark surround should also be addressed. In this study, all windows in the experimental room needed to be sealed. Future work exploring the use of head- 
mounted display with tight seal should be conducted to improve the usability of this method.

\section{ACKNOWLEDGMENT}

This study is partially supported by the Hong Kong Research Grants Council through GRF grant 619907.

\section{REFERENCES}

[1] Aschan, G., and M. Bergstedt (1955), The genesis of secondary nystagmus induced by vestibular stimuli. Acta Sot. Med. (Upsala) 60: 113.

[2] Cheung BS, Howard IP, Money KE (1991), Visually induced sickness in normal and bilaterally labyrinthine-defective subjects. Aviat Space Environ Med 62:527-531.

[3] Chow, E.H.C. (2008) Postural disturbance and vection when viewing visual stimulus oscillating in roll and fore-and-aft directions: effects of frequency and peak velocity. Unpublished thesis, the Hong Kong University of Science and Technology.

[4] Chow, E.H.C., Ji J.T.T., So R.H.Y. and Cheung, R.T.F. (2007) The Effect of Visual Stimulus Oscillation Frequency on Postural Disturbance in Roll and in Fore-and-Aft Direction. Proceedings of the First International Symposium on Visually Induced Motion Sickness, Fatigue, and Photosensitive Epileptic Seizures (VIMS2007), 10-12 Dec., Hong Kong. pp.165-170.

[5] Cohen B, Matsuo V, Raphan T (1977), Quantitative analysis of the velocity characteristics of optokinetic nystagmus and optokinetic after-nystagmus. J Physiol (Lond) 270: 321-344.

[6] Cohen B, Uemura T, Takemori S. (1973) Effects of labyrinthectomy on optokinetic nystagmus (OKN) and optokinetic after-nystagmus (OKAN). Int J Equilib Res. 1973 Jun 3(1): 88-93.

[7] Cohen B, Dai M, Raphan T (2003) The critical role of velocity storage in production of motion sickness. Ann NY Acad Sci 1004:359-376.

[8] Dai M, Kunin M, Raphan T, Cohen B (2003) The relation of motion sickness to the spatial-temporal properties of velocity storage. Exp Brain Res 151:173-189.

[9] Dai M, Raphan T, Cohen B (2005) Effects of baclofen on the angular vestibulo-ocular reflex. Exp Brain Res 1-10.

[10] Dai M, Raphan T, Cohen B (2007), Labyrinthine lesions and motion sickness susceptibility, Exp Brain Res 178:477-487.

[11] Dellepiane, M., Medicine, M.C., Barettini, L., Mura, A.C. (2006) Correlation between vestibulo-ocular reflex and optokinetic afternystagmus in normal subjects and in patients with vestibular system disorders, ACTA OTORHINOLARYNGOL ITAL 26: 20-24.

[12] Ebenholtz SM (1992) Motion sickness and oculomotor systems in virtual environments, Teleoperators and Virtual Environments 1(3), 302-305.

[13] Golding (2006) Predicting individual differences in motion sickness susceptibility by questionnaire, Personality and Individual Differences 41: 237-248.

[14] Guo, C.T., Ji, J.T.T. and So, R.H.Y. (2011) Correlations between time constants of decaying OKAN slow phase velocity and rated levels of visually induced motion sickness. Awaiting publication.

[15] Hu S, Stern RM, Vasey, M.W. and Koch, K.Ll (1989), Motion sickness and gastric myoelectric activity as a function of speed of rotation of a circular vection drum. Aviat Space Environ Med. May, 60(5): $411-4$

[16] Ji, J.T.T., So, R.H.Y. and Cheung, R.T.F. (2009) Isolating the Effects of Vection and Optokinetic Nystagmus on Optokinetic Rotation-Induced Motion Sickness. Human Factors, 51(5), pp.739751.

[17] Kennedy, R.S., Lane, N.E., Berbaum, K.S., and Lilienthal, M.G. (1993). A simulator sickness questionnaire (SSQ): A new method for quantifying simulator sickness. International Journal of Aviation Psychology, 3, 203-220.

[18] Kiryu, T. and So, R.H.Y. (2008) Sensation of Presence and Cybersickness in applications of Virtual Reality for Advanced Rehabilitation. Journal of NeuroEngineering and Rehabilitation, 4:34.

[19] Lo, W.T. and So, R.H.Y., (2001) Cybersickness in the presence of scene rotational movements along different axes, Applied Ergonomics, Vol.32, No.1, 2001, pp. 1-14.

[20] Maioli, C. (1988), Optokinetic Nystagmus: Modeling the Velocity Storage Mechanism, The Journal of Neuroscience 8(3): 821-832.

[21] Muratore R. and Zee, D.S. (1979), Pursuit afternystagmus, Brain Research 19: 1057-1059.

[22] Ohm, J. (1927) Zur Augenzitterkunde. Mitteilung. Der optische Drehnachnystagmus. Albrecht Von Graefes Arch. Klin. Ophthalmol. 118: 103-1 17.

[23] So, R.H.Y. and Griffin, M.J. (1991) Effects of time delays on head tracking performance and the benefits of lag compensation by image deflection. Proceedings of Flight Simulation Technologies Conference, New Orleans, Louisiana, 12-14 August, 1991. American Institute of Aeronautics and Astronautics, Aerospace Center, Washington, AIAA-91-2926-CP, 124-130.

[24] So, R.H.Y. and Griffin, M.J. (1992), Compensating lags in headcoupled displays using head position prediction and image deflection. J. Aircraft. 29(6): 1064-1068, 1992.

[25] So, R.H.Y. and Griffin, M.J. (2000) Effects of target movement direction cue on head-tracking performance. Ergonomics, Vol.43, No.3, pp. 360-376.

[26] So, R.H.Y. and Lo, W.T. (1999) Cybersickness: An Experimental Study to Isolate the Effects of Rotational Scene Oscillations." Proceedings of IEEE Virtual Reality '99 Conference, March 13-17, 1999, Houston, Texas. Published by IEEE Computer Society, pp.237-241.

[27] So, R.H.Y. and Ujike, H. (2010) Visually induced motion sickness, visual stress and photosensitive epileptic seizures: what do they have in common? - Preface to the special issue. Applied Ergonomics, 41(4), pp.491-493.

[28] So, R.H.Y., Finney, C.M. and Goonetilleke, R.S. (1999) Motion sickness susceptibility and occurrence in Hong Kong Chinese. Contemporary Ergonomics 1999, Taylor \& Francis, pp.88-92.

[29] So, R.H.Y., Ho, A.T.K. and Lo, W.T. (2001) A proposed metric to quantify virtual scene movement for the study of cybersickness: definition, implementation and verification". PRESENCE, 10, pp. 193-216.

[30] So, R.H.Y., Lo, W.T. and Ho, A.T.K., (2001) Effects of navigation speed on motion sickness caused by an immersive virtual environment. Human Factors, 43, 2001, pp.452-261.

[31] Steele, J, E. (1968) The symptomatology of motion sickness. In fourth symposium on the role of the

[32] Takahashi M, Ogata M, Miura M. (1997). The significance of motion sickness in the vestibular system, J Vestib Res. 7(2-3): 1

[33] Tijssen, M.A.J, Straathof, C.M., Hain, T.C. and See, D.C. (1989), Optokinetic afternystagmus in humans: normal values of amplitude, time constant, and asymmetry, Ann Otol Rhinol Laryngol 98.

[34] Waespe, W., Cohen, B. and Raphan, T. (1983), Role of the flocculus and paraflocculus in optokinetic nystagmus and visual-vestibular interactions: Effects of lesions 50: 9-33. 